\title{
Design of English Remote Education System Based on GPRS Network
}

\author{
XIAO Yan ${ }^{1, a}$, LI Ying ${ }^{1, b}$ \\ ${ }^{1}$ Qingdao Huanghai College, China \\ a1853346016@qq.com, bxiaoyan82313@163.com
}

\begin{abstract}
Keywords: GPRS; remote communication; English teaching; system design
Abstract. Along with economic development, English has been playing an increasingly important role in each industry. The remote education relies on the Internet, so, communication in areas can't be achieved without network coverage. Not only the English teaching ability and its flexibility can be improved effectively but also the problem of distance teaching between teachers and students can be solved through the design and research on remote English teaching system based on the GPRS network. The system takes the form of the "heartbeat packet" as data interval which is consistent with the GPRS terminal connection and online to enhance the communication capability.
\end{abstract}

\section{Introduction}

With the economic development and internationalization, the fields of both social various industries put forward higher requirements on English and only a variety of teaching models can meet the needs of different groups of people[1]. Remote English Teaching can use advanced communication technology conditions, such as the Internet, to solve the problem of long distance teaching to some extent. However, in some areas, especially in remote areas, broadband coverage is very low, which can not be achieved in the distance teaching. With the development of GPRS technology, the network type has been developed from $2 \mathrm{G}$ to $5 \mathrm{G}$, and the coverage rate is very wide, which provides a good carrier for distance education. It can be seen in this paper that with the research on the remote English teaching system based on the GPRS network, English distance education is no longer limited to the Internet. It is of great significance to the promotion of English education and teaching quality.

\section{System Design Requirements}

Modern distances of education throughout the world can meet the needs of different levels of people. In universities, distance education has created opportunities for more people to receive higher education. In the company, distance education can be used for staff skills training so that they adapt to the progress of technology. For individuals, through distance education to accept vocational skills training, they can create more employment opportunities for themselves; for the government, the use of distance education online teacher training, it can improve the quality of teaching in primary and secondary schools. And for remote areas, distance education has solved the difficult problem of teaching[2]. Enrichment of digital resources and curriculum offers a variety of selective for people's lifelong learning. The development of the specialized team of distance education has been adapted to the needs of the development of the scale. The construction of the system of distance education has already begun and the information infrastructure has created the basic conditions for the distance education.

Each functional module of the system is relatively independent and mutually influenced. So it is an organic whole. Each functional module is provided to different users who are with each other to meet their different needs. For example, the system set the class department and other agencies, set the administrators, teachers and students and other users, in addition to courses and courseware and work and so on. They need to have a comprehensive management of these users, courses and resources belong to which institutions and the user has what kind of resources[3]. 
In the long distance English teaching system, in order to achieve, enrich and expand the teaching means, data transmission and communication are needed in addition to audio and video, there are a number of specific instructions. The data transmission of system mainly depends on the GPRS wireless network. In the process of communication, it is necessary to complete the protocol functions of the link layer, network layer and transport layer and the fundamental modules is GR47 wireless module. In the module, between the data link layer applied point to point protocol and transport layer using the TCP protocol[4].

\section{System Design}

Hardware Design. In order to carry out a better teaching management, the system uses the MSP130F149 microcontroller as the main control unit as shown in Fig.1. Among them, the ground pin and the power supply provide the input port for the external clock and crystal oscillator and output $1 / 4$ fuse in RC oscillation mode. The reset pin of the clock is MCLR, which generally can be controlled manually and it is effective at low power. I/O pin has a very high running speed. Timer/counter has two kinds of 16 and 8 bits and they all can in normal work under the standby mode.

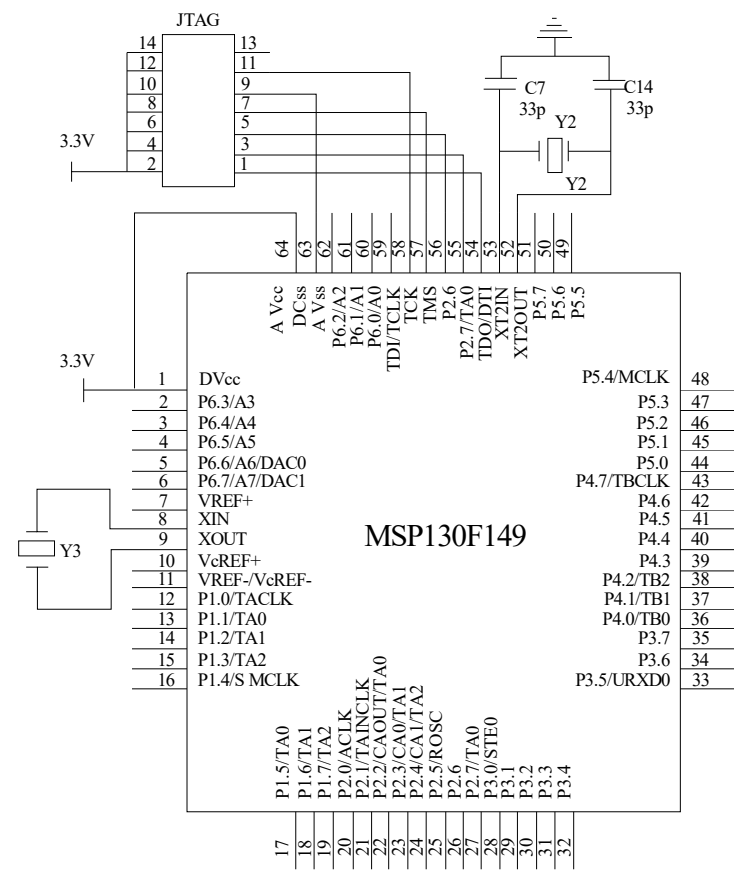

Fig.1 The wiring diagram of single chip microcomputer

In this paper, RS485 is used as the transmission protocol. Its characteristics are high transmission rate and anti-interference ability. With a low interface level, it is not easy to damage the chip[5]. This kind of protocol can support more nodes and its transmission distance is far. The interface circuit diagram of RS485 is shown in Fig.2.

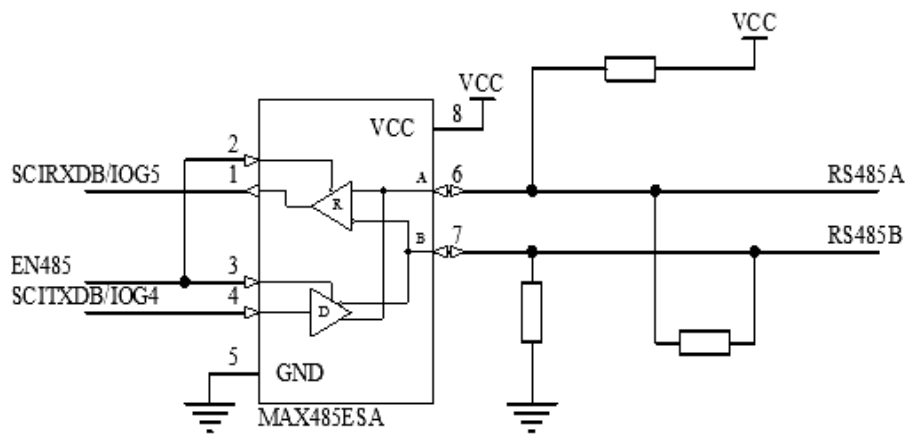

Fig. 2 The serial communication interface circuit

In the process of implementing network communication function, firstly, power on and initialize the GR47 wireless module to ensure that the normal function of the module. Secondly, apply the 
point-to-point call service through the point-to-point protocol negotiations with GGSN of China Mobil. Establish the point-to-point link to realize the transmission of network data by sends a connection request to terminal system from a GR47 module. Finally, through the IP, TCP, PPP data package, data transmission is completed. In the process of data transmission, based on the Socket connection, the data is sent to the GR47 wireless module through the serial port in near-real time, and then be converted and packaged based on the relevant agreement by the GR47 wireless module.

Software Design.In the software control, the design of a database is one of the most key factors that affect the control effect. As shown in Fig.3, DBMS is connected with the database to set the local interface, so DBMS can be very easy to access the data in the database and ensure the normal work of the application subroutine[6]. It is necessary that consider the factors in many aspects of the storage format and functional requirements in the database design because the database as the storage of data and instructions in the virtual container and through a full range of analysis to reduce the development cycle. Therefore, in the design of the database, the first step is to analyze the specific requirements of the entire system, working principles and processes so as to better complete the task of guiding the work.

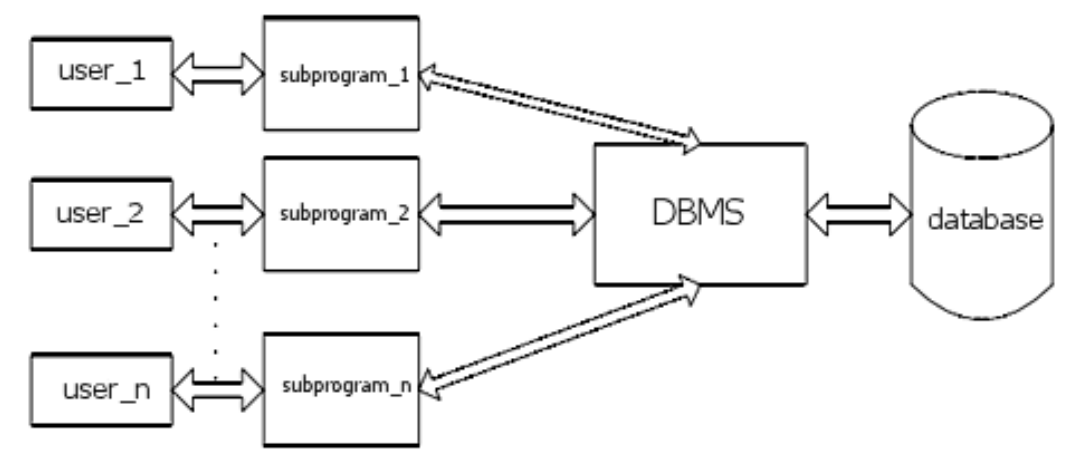

Fig.3 Database workflow

In this paper, the database is design in the form of data table which build state information as the center of the database. The information tables as shown in Fig.4.

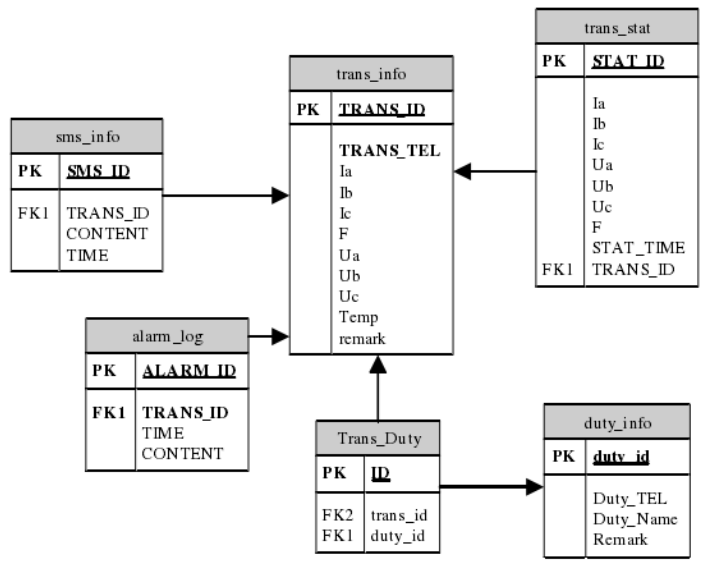

Fig.4 Database tables

\section{Improvement of Communication Ability}

The normal operation of the GR47 wireless module can be controlled through the transmission of the AT command. It has entered the command mode after connect GR47 module into the GPRS network. Through the microcontroller issued commands command. GR47 module completes the corresponding network operation. Once detection system into the instruction of grammatical errors and GR47 module will immediately send a bug report through the serial port. When the instruction is all normal, the connection status is returned to the server. GPRS for automatic system for distribution of a dynamic IP address when data transmission system attached to the GPRS network. Due to the IP address is the address is fixed within the network address, it can't be achieved in an external 
communication. At this time, the AT instruction will get local IP address according to the corresponding format is sent to the monitoring center of the IP address and port, and you can achieve data exchange between each other.

When the AT command is issued, the GR47 module will enter the data mode and no response to AT instruction execution of arbitrary operation. In the data mode, the data transmission rate is largely determined by the serial transmission rate and the strength of the GPRS network signal. The GR47 module mainly sends data packets to the IP package through the serial port. And transmit point to point protocol layer through specific IP port. The attached network of GPRS and Flexible phase online applied to the stage of point-to-point protocol. Among them, through the integration of GR47 module and embedded TCP/IP protocol stack, the network and flexible access can complete through the processor's command control function independently and do not need to manually set. AT the transmission from the serial interface instructions can make the system to establish a TCP connection and control GR47 module to perform online functions.

In general, there will be some server disconnection if no data transmission after a period of time when completed in the GR47 module of GPRS network connection. Namely the problem which GPRS network data transmission line causes data sending is suspended. In order to solve this problem, the commonly used method is sending. It keeps the GPRS terminal connection and online through the "heartbeat packet" data every few minutes. In practice, it can apply for VPN virtual private network through the mobile network access business or apply for a SIM card and IP address binding. In network communication based on GPRS, the problem of data packet dropout is relatively rare. And for the improvement of the reliability of the monitoring terminal, it can ensure the stability and security of data transmission through data retransmission.

\section{Conclusions}

The distance education system has made up for the shortage of education funds and has accelerated the level of English education in various industries and fields and has met the requirements of national economic development. With the degree of information technology in China, more people can complete the teaching and learning of English through mobile network. It has created a new way of distance learning and become the focus of research in the field of education. As a dedicated learning platform, you can achieve communication between teachers and students at any time because of the convenience of GPRS wireless network.

\section{References}

[1] L.P. XIAO: Journal of The Chinese Society of Education, Vol(2002)No.5,p33-35.

[2] Y.SH. MA: Journal of Hebei University of Science \& Technology,Vol(2010) No24,p20-23.

[3] Y.Q. HU: Proceedings of the CSEE,Vol(2003)No23,p137-140.

[4] B.LI: Mechanical Engineering \& Automation,Vol(2015)No4,p78-81.

[5] Sh.R.Wang, Ch.H.Yao: Chinese Foreign Language, Vol(2013)No.5,p4-10.

[6] R. Kai: Journal of Nanjing Normal University,Vol(2009) No9,p18-21. 